\title{
Fingerprints of the Anisotropic Spin-Split Hole Dispersion in Resonant Inelastic Light Scattering in Two-Dimensional Hole Systems
}

\author{
M. Hirmer, ${ }^{1}$ M. Hirmer, ${ }^{1}$ D. Schuh, ${ }^{1}$ W. Wegscheider,${ }^{2}$ T. Korn, ${ }^{1}$ R. Winkler, ${ }^{3}$ and C. Schüller ${ }^{1, *}$ \\ ${ }^{1}$ Institut für Experimentelle und Angewandte Physik, Universität Regensburg, D-93040 Regensburg, Germany \\ ${ }^{2}$ Solid State Physics Laboratory, ETH Zurich, 8093 Zurich, Switzerland \\ ${ }^{3}$ Department of Physics, Northern Illinois University, DeKalb Illinois 60115, USA
}

(Received 14 June 2011; published 18 November 2011)

\begin{abstract}
In resonant inelastic light scattering experiments on two-dimensional hole systems in GaAs- $\mathrm{Al}_{x} \mathrm{Ga}_{1-x} \mathrm{As}$ single quantum wells we find evidence for the strongly anisotropic spin-split hole dispersion at finite in-plane momenta. In all our samples we detect a low-energy spin-density excitation of a few meV, stemming from excitation of holes of the spin-split ground state. The detailed spectral shape of the excitation depends sensitively on the orientations of the linear light polarizations with respect to the inplane crystal axes. In particular, we observe a doublet structure, which is most pronounced if the polarization of the incident light is parallel to the [110] in-plane direction. Theoretical calculations of the Raman spectra based on a multiband $\mathbf{k} \cdot \mathbf{p}$ approach confirm that the observed doublet structure is due to the anisotropic spin-split hole dispersion.
\end{abstract}

PACS numbers: 73.20.Mf, 73.21.Fg, 73.61.Ey, 78.30.-j

Recently, the spin physics of two-dimensional hole systems (2DHSs) in $p$-doped semiconductor systems has gained interest in semiconductor spintronics research [1], because surprisingly long spin dephasing times of holes, localized in potential fluctuations in GaAs-AlGaAs quantum wells (QWs) [2-4], of up to several tens of nanoseconds were reported [4]. Apart from Zeeman spin splitting in an external magnetic field $B$, charge carriers, confined in QWs of zincblende-type material, exhibit a spin splitting due to relativistic spin-orbit (SO) coupling for finite in-plane momenta $\mathbf{k}_{\|}$, even at $B=0$ [5]. The SO coupling originates from the bulk inversion asymmetry (Dresselhaus effect) [6], and, if the confinement potential is asymmetric, from the so-called structure inversion asymmetry (Rashba effect) [7]. In QWs with an asymmetric potential profile, the spin splitting of holes [8] due to the Rashba SO coupling is often dominant over the Dresselhaus contribution [5]. In general Rashba splitting is anisotropic, following the cubic symmetry of the underlying crystal structure (point group $C_{4 v}$ for a (001) surface [9]), resulting in a fourfold splitting pattern. If both Rashba and Dresselhaus SO coupling are present, the symmetry is reduced to $C_{2 v}$, resulting in a twofold splitting pattern with main axes [110] and [110].

Over the past three decades, resonant inelastic light scattering (RILS) has proven to be a highly valuable tool for the investigation of electronic excitations in semiconductor nanostructures [10]. In spontaneous RILS, real electronic excitations are created in the system, by taking advantage of resonance enhancements of the process by virtual resonant intermediate states. Because of polarization selection rules, the different types of collective excitations of electrons can be distinguished in RILS experiments: For parallel polarizations of incident and scattered photons (so-called polarized geometry) chargedensity excitations (CDEs) can be observed, and for crossed polarizations (depolarized geometry) spin-density excitations (SDEs) are allowed. For 2DHSs in GaAs QWs it was found that many-particle interactions are less important than for electron systems (2DESs) [11], and the energies of hole excitations can be described approximately by single-particle transitions, based on $\mathbf{k} \cdot \mathbf{p}$ band-structure calculations $[12,13]$. In a pioneering work, Jusserand et al. observed the anisotropic $B=0$ spin splitting of 2DESs in GaAs QWs by RILS [14,15] reflecting the interplay of Rashba and Dresselhaus SO coupling that are often of similar magnitude in 2DESs. Here we present RILS experiments on high-mobility 2DHSs in asymmetric GaAs-AlGaAs single quantum wells (SQWs). We identify a low-energy SDE, which we attribute to an excitation of the spin-split hole ground state. We show that the spectral shape of this excitation depends on the relative orientations of incident and scattered light polarizations with respect to crystallographic axes. In particular, we show that there are preferential in-plane directions, where a pronounced splitting of the SDE can be observed. By theoretical modeling we can show that this splitting is a fingerprint of the anisotropic spin-split hole dispersion of the 2DHS.

Our samples are one-sided carbon $p$-doped GaAs- $\mathrm{Al}_{0.3} \mathrm{Ga}_{0.7} \mathrm{As}$ SQWs with well widths of $15 \mathrm{~nm}$, $20 \mathrm{~nm}$, and $25 \mathrm{~nm}$. Series of samples with different spacer widths between QW and doped barrier region of $20 \mathrm{~nm}$, $15 \mathrm{~nm}$, and $10 \mathrm{~nm}$ were grown in [001] direction by molecular beam epitaxy. The different spacer widths result in different hole densities between about $p=$ $4 \times 10^{11} \mathrm{~cm}^{-2}$ and $p=9 \times 10^{11} \mathrm{~cm}^{-2}$ at $T=4.2 \mathrm{~K}$, and hole mobilities of up to $\mu_{h}=5 \times 10^{5} \mathrm{~cm}^{2} / \mathrm{V} \mathrm{s}$. In the following we concentrate on two samples with $20 \mathrm{~nm}$ 
and $25 \mathrm{~nm}$ QW widths and a $10 \mathrm{~nm}$ spacer. In these samples with the largest hole densities the observed effects are most pronounced. The RILS experiments were performed in quasibackscattering geometry at liquid He temperatures. For excitation, a cw Ti:sapphire laser was used, and the signals were detected by a triple Raman spectrometer and a CCD camera. For comparison, the band structure and dipole matrix elements were calculated based on an $8 \times 8$ Kane Hamiltonian using the program package Nextnano $^{3}$ [16].

First, we discuss the main idea of the experiments. Figure 1(a) shows the typical asymmetric valence-band profile, calculated for the $20 \mathrm{~nm}$ SQW sample with a $10 \mathrm{~nm}$ spacer. Energy and charge density of the ground state are indicated. The confining potential seen by the 2DHS is highly asymmetric, thus resulting in a significant Rashba contribution to the SO interaction. In Fig. 1(b), the $B=0$ spin splitting of the heavy-hole (hh) ground state for finite in-plane momentum $k_{\|}$is shown (labeled $\mathrm{hh}_{0}^{-}$and $\mathrm{hh}_{0}^{+}$) together with the calculated dispersion of the first two
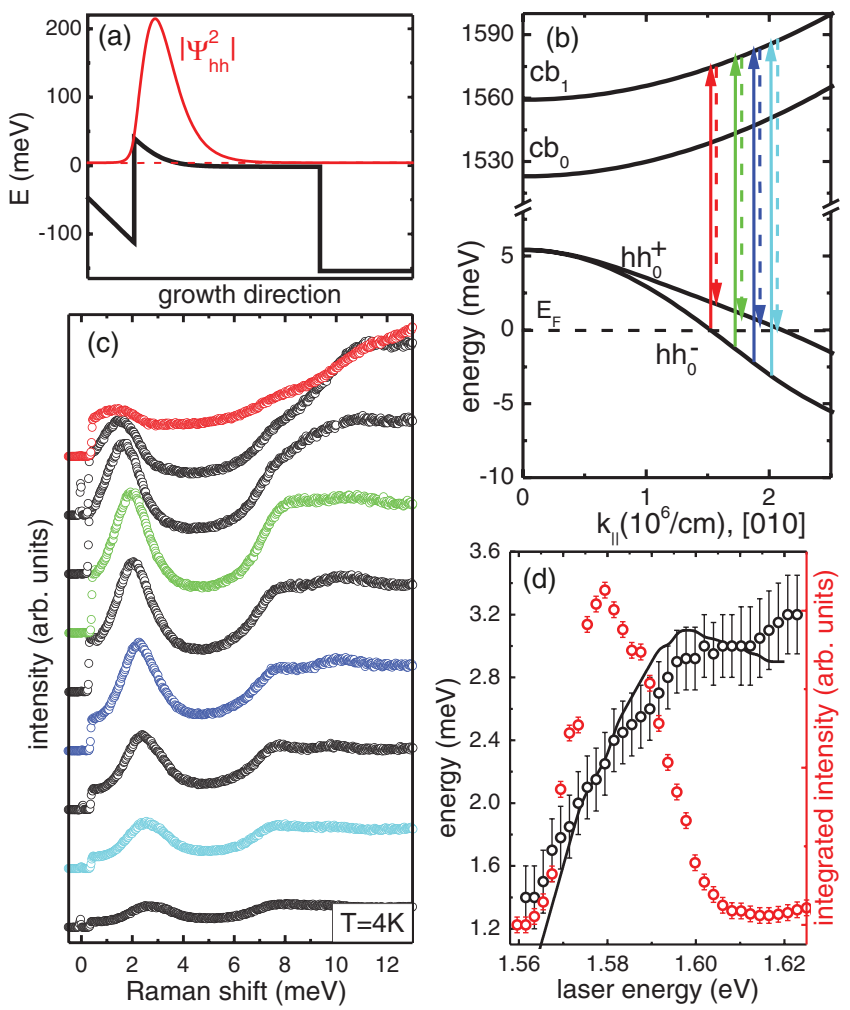

FIG. 1 (color). (a) Valence-band potential profile of a $20 \mathrm{~nm}$ SQW with hole density $p=8.6 \times 10^{11} \mathrm{~cm}^{-2}$. (b) Spin-split dispersion of the $\mathrm{hh}_{0}$ subband and the lowest two conductionband subbands. (c) A series of depolarized $\bar{z}([010]$, [100])z RILS spectra for different laser energies from $1.567 \mathrm{eV}$ to $1.600 \mathrm{eV}$ (top to bottom spectra). Spectra are taken at nominally $T=4 \mathrm{~K}$. (d) Experimental mode positions and integrated intensities of the low-energy SDE. The solid line represents the mode position, as extracted from calculated RILS spectra. conduction-band subbands (labeled $\mathrm{cb}_{0}$ and $\mathrm{cb}_{1}$ ). The spin splitting of the conduction subbands is much smaller $(<0.11 \mathrm{meV}$ in the displayed range), and not resolved in this plot. In the RILS experiment, in the first step $a h_{0}^{-}-\mathrm{cb}_{1}$ electron-hole pair is created by resonant absorption of a photon [solid arrows in Fig. 1(b)]. In the second, instantaneous step, the photoexcited electron recombines with a hole in the $\mathrm{hh}_{0}^{+}$state above the Fermi energy $E_{F}$ [dashed arrows in Fig. 1(b)]. As a net effect, $\mathrm{a} \mathrm{hh}_{0}^{+} \rightarrow \mathrm{hh}_{0}^{-}$spin-flip hole excitation is created within the spin-split hh ground state. We emphasize that, in contrast to luminescence, steps 1 and 2 are instantaneous in RILS. By choosing different wavelengths of the exciting laser (indicated by different colors of vertical arrows), we selectively boost the contribution of specific regions in $k_{\|}$space to the RILS signal due to resonance enhancements. More specifically, transitions, starting from a constant-energy contour of the $\mathrm{hh}_{0}^{-}$state in the $k_{x}-k_{y}$ plane dominantly contribute to the spectrum for a given laser energy.

Next, we discuss the representative experimental data. Figure 1(c) shows depolarized RILS spectra for different laser energies. The laser photon energies increase from top to bottom spectra from $1567 \mathrm{meV}$ to $1600 \mathrm{meV}$. The experimental situations for the selected, colored spectra in Fig. 1(c) correspond roughly to the colored arrows in Fig. 1(b) of the same color (conduction-band subband $\mathrm{cb}_{1}$ as intermediate state). A pronounced peak can be observed in the spectra at a Raman shift of about 1 to $3 \mathrm{meV}$ in Fig. 1(c). Specifically, this excitation is visible in depolarized spectra, for crossed polarizations of incident and scattered light, only, and it is at least 1 order of magnitude weaker in the polarized configuration (not shown). We interpret this excitation as a SDE of the spin-split hh ground state (in the following referred to as R-SDE), as explained above. The signals for Raman shifts larger than about $7 \mathrm{meV}$ in Fig. 1(c) are due to the onset of the continuum of hole intersubband transitions to higher hole subbands [17]. Remarkably, these signals are present in both polarization configurations, since to higher subbands, both, CDEs (non-spin-flip transitions) and SDEs (spin-flip transitions) are possible [12,13]. Figure 1(d) shows a more detailed analysis of the energy of the R-SDE in dependence on laser photon energy. Indeed, an increase of the energy of the R-SDE can be seen with increasing laser energy that resembles the increasing hole spin splitting with increasing in-plane momentum $k_{\|}$[cf. Figure 1(b)]. The solid line represents the calculated mode position for comparison. The calculated RILS spectra, which are summed over all $\mathbf{k}_{\|}$space, are described below in more detail. The integrated intensity of the R-SDE exhibits a pronounced resonance with its maximum around $1580 \mathrm{meV}$ laser energy.

Now, we come to the central feature of our investigations. In our experiments on 2DHS we have detected for the first time a distinct dependence of the spectral shape on polarization orientations with respect to the in-plane 
crystal axes. Figures 2(a)-2(c) show depolarized spectra in the vicinity of the resonance maximum [Fig. 2(a)], and for laser energies above the maximum [Figs. 2(b) and 2(c)]. The four spectra in each panel are taken for different depolarized polarization configurations $\bar{z}(a, b) z$, as indicated in Fig. 2. Here, $\bar{z} \|[00 \overline{1}]$ and $z \|[001]$ are the directions of the incoming and scattered photons, respectively. $a$ and $b$ indicate the directions of the linear light polarizations of the incoming and scattered light, respectively. A double peak structure is clearly visible, which is most pronounced in the scattering geometry $\bar{z}([110],[1 \overline{1} 0]) z$, i.e., with the laser polarization parallel to the [110] in-plane direction, and least pronounced in the $\bar{z}([1 \overline{1} 0],[110]) z$ configuration. The spectra of the other two configurations, with polarizations parallel to the principal crystal axes [100] and [010], are very similar, as expected by symmetry [9]. We interpret the doublet structure to be caused by the anisotropic spin-split hole dispersion of the hh ground state within the $k_{x}-k_{y}$ plane.

As a first evidence, we compare the observed peak positions with the calculated spin splittings at the Fermi energy, at a constant energy of the $\mathrm{hh}_{0}^{-}$state [cf. vertical arrows in Fig. 2(d)], along the most different [010] and [110] in-plane directions in Fig. 2(d). As can be seen, the splittings are markedly different in these two directions and
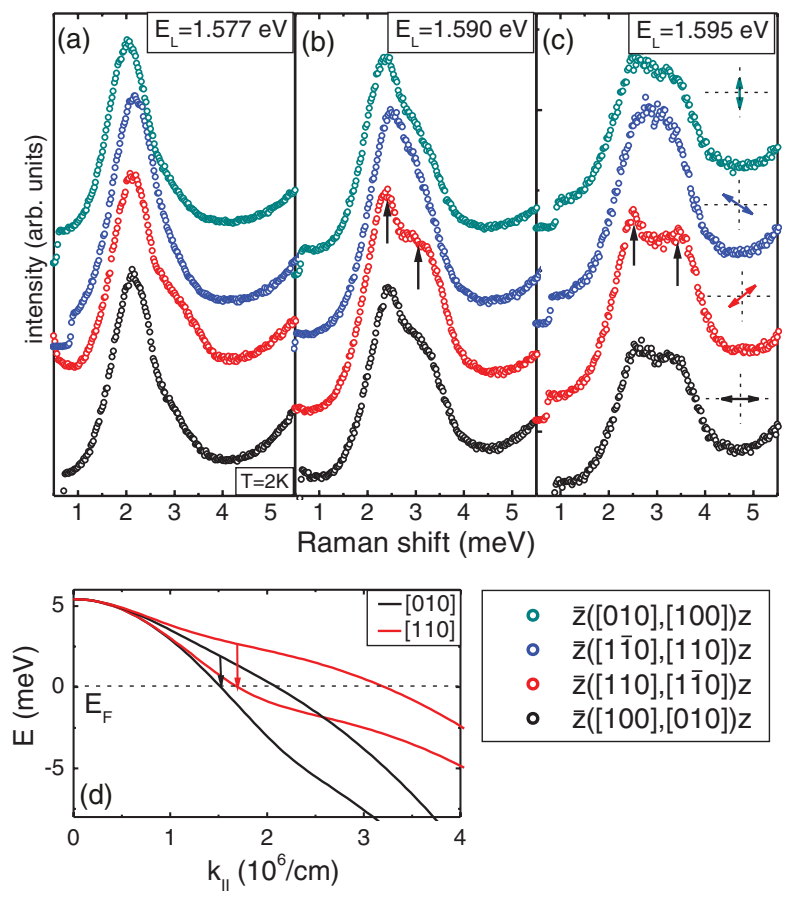

FIG. 2 (color). (a)-(c) Depolarized RILS spectra of the $20 \mathrm{~nm}$ SQW sample for different scattering geometries as indicated in the legend, and for three different laser photon energies $E_{L}$. Orientation of laser polarization is indicated on the right. Spectra are taken at $T=2 \mathrm{~K}$. (d) Calculated spin-split dispersion of the $\mathrm{hh}_{0}$ subband for the $20 \mathrm{~nm} \mathrm{SQW}$ for two different in-plane directions. Transitions, which may contribute to the R-SDE, are indicated by arrows. of similar magnitude as the positions of the experimentally observed doublet structure. Of course, for a thorough analysis, we have to consider resonant transitions as shown in Fig. 1(b), and sum over all $\mathbf{k}_{\|}$space in order to calculate RILS spectra. Consequently, we expect the spectral shapes to depend sensitively on the specific resonant intermediate state in the conduction band, since the transition probabilities should generally be different for different intermediate states. To highlight this further, Fig. 3 shows selected experimental spectra of a $25 \mathrm{~nm} \mathrm{SQW}$ for resonance with $\mathrm{cb}_{1}$ [Fig. 3(a)] and $\mathrm{cb}_{2}$ subbands [Fig. 3(b)] as intermediate states. The laser photon energies increase from top to bottom spectra. Here, we have chosen the $25 \mathrm{~nm}$ SQW sample, since in this wider well we can easily reach the $\mathrm{cb}_{2}$ resonance within the tuning range of our laser. The spectra are taken in the $\bar{z}([110],[1 \overline{1} 0]) z$ configuration, where the doublet structure of the R-SDE is most pronounced. First, we can see that the overall line shape of the R-SDE for the resonance with the $\mathrm{cb}_{1}$ subband [Fig. 3(a)] for this scattering geometry is very similar to the R-SDE in the $20 \mathrm{~nm}$ SQW (cf. red spectra in Fig. 2). It can be seen in Fig. 3(a) that the higher-energy component of the doublet in the spectra (marked with red arrows) appears as a high-energy shoulder for all laser photon energies. However, for the $\mathrm{cb}_{2}$

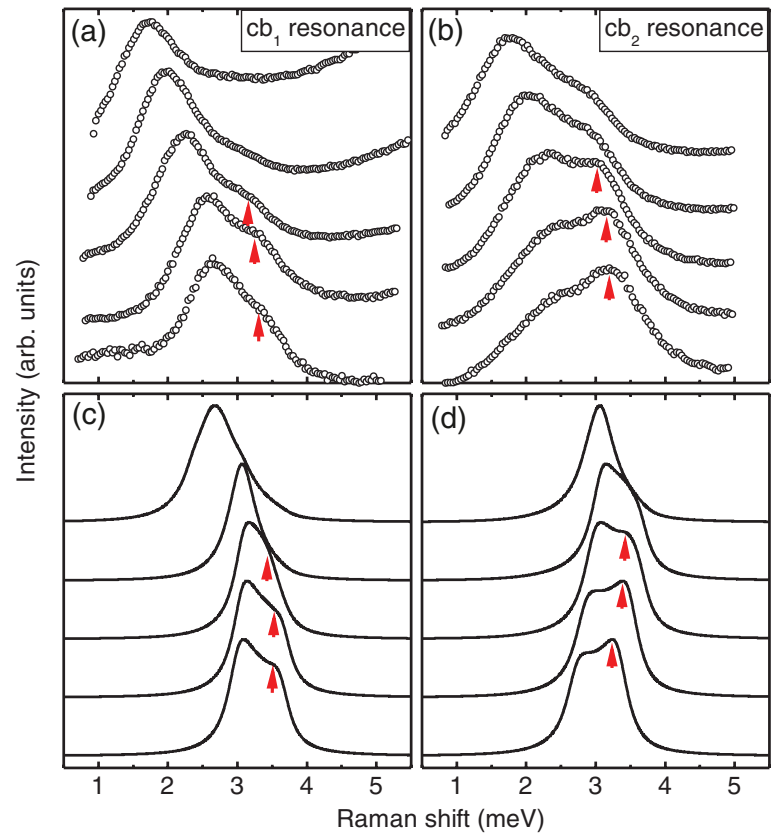

FIG. 3 (color). Depolarized $\bar{z}([110],[1 \overline{1} 0]) z$ RILS spectra of the $25 \mathrm{~nm}$ SQW sample with hole density $p=8.4 \times 10^{11} \mathrm{~cm}^{-2}$ for (a) resonance with conduction-band subband $\mathrm{cb}_{1}$, laser photon energies increase from top to bottom spectra from $1.554 \mathrm{eV}$ to $1.577 \mathrm{eV}$, and, (b) with subband $\mathrm{cb}_{2}$, laser photon energies increase from top to bottom spectra from $1.590 \mathrm{eV}$ to $1.614 \mathrm{eV}$. Spectra are taken at $T=2 \mathrm{~K}$. (c) Calculated RILS spectra for the same parameters as in the experiments in Fig. 3(a), and, (d) as in the experiments in Fig. 3(b). All spectra are normalized and shifted vertically for better comparison. 
resonance [Fig. 3(b)], the shoulder becomes dominant at higher laser energies, which will be investigated in more detail below.

To validate our interpretation of the observed doublet structure of the R-SDE to be due to the anisotropic hole spin splitting, we have modeled RILS spectra using multiband $\mathbf{k} \cdot \mathbf{p}$ calculations based on the $8 \times 8$ Kane Hamiltonian. If many-particle interactions are neglected, the RILS intensity $I\left(\omega_{i}, \omega\right)$ for scattering on single-particle excitations is approximately given by $[13,18]$

$$
\begin{aligned}
I\left(\omega_{i}, \omega\right) \propto & \operatorname{Im}\left\{\sum_{\mathbf{k}_{\|}}\left|\sum_{c} \frac{\left\langle\beta\left|\mathbf{e}_{s} \cdot \mathbf{p}\right| c\right\rangle\left\langle c\left|\mathbf{e}_{i} \cdot \mathbf{p}\right| \alpha\right\rangle}{E_{\alpha}\left(\mathbf{k}_{\|}\right)-E_{c}\left(\mathbf{k}_{\|}\right)+\hbar \omega_{i}-i \Gamma}\right|^{2}\right. \\
& \left.\times\left[\frac{f_{\alpha}-f_{\beta}}{\hbar \omega+E_{\alpha}\left(\mathbf{k}_{\|}\right)-E_{\beta}\left(\mathbf{k}_{\|}\right)-i \gamma}\right]\right\} .
\end{aligned}
$$

For our specific case, $\alpha$ and $\beta$ are the $\mathrm{hh}_{0}^{-}$and $\mathrm{hh}_{0}^{+}$states, respectively, and $c$ labels the two spin states of the conduction subband $\mathrm{cb}_{i}$, which serve as intermediate states in the RILS process [cf. Figure 1(b)]. $\mathbf{e}_{i}\left(\mathbf{e}_{s}\right)$ is the polarization vector of the incident (scattered) photons, and, $\Gamma$ and $\gamma$ are phenomenological broadening constants of the resonance transition and the hole excitation, respectively. $\hbar \omega_{i}$ is the energy of the laser photons and $f_{i}$ are the Fermi distribution functions of the hole subbands. In Eq. (1) we have considered the most resonant case in the scattering amplitudes, only. The sum over $\mathbf{k}_{\|}$is numerically performed on a grid, taking into account the Fermi functions and the warping of the spin subbands. Figures 3(c) and 3(d) show spectra, which are calculated corresponding to the parameters (laser energies and temperature) of the experimental spectra in Figs. 3(a) and 3(b), respectively. Owing to the simplifications in our model, the comparison can be semiquantitative, only. Nevertheless, the doublet structure is clearly reproduced, as well as the right order of magnitude of the excitation energies and splittings of the experimental R-SDE. In particular, also the stronger relative weight of the higher-energy component of the doublet for the $\mathrm{cb}_{2}$ resonance [red arrows in Figs. 3(b) and 3(d)] are reproduced in the theoretical curves. Because of the resonance enhancements, excitations starting at a constantenergy contour of the $\mathrm{hh}_{0}^{-}$state contribute dominantly to the RILS spectrum at a given laser energy. These resonant transitions are weighted by the $\boldsymbol{k}_{\|}$and polarization dependent dipole matrix elements, thus giving rise to a double peak structure reflecting the anisotropic spin-split hole dispersion.

We note that the calculations of RILS spectra are based on a $\mathbf{k} \cdot \mathbf{p}$ model neglecting bulk inversion asymmetry. Hence, the calculated constant-energy contours in the $k_{x}-k_{y}$ plane show the typical fourfold symmetry for this case. Consequently, calculated spectra for $\bar{z}([110],[1 \overline{1} 0]) z$ and $\bar{z}([1 \overline{1} 0],[110]) z$ are equal. A close inspection of the spectra in Fig. 2 evidences differences in the line shapes of the R-SDE observed in these two scattering configurations (red and blue spectra in Fig. 2). This indicates that, indeed, the symmetry of constant-energy contours in the $k_{x}-k_{y}$ plane is reduced from fourfold to twofold, which reflects the interplay of Rashba and Dresselhaus SO coupling [9].

In conclusion, we have identified a low-energy SDE in one-sided $p$-doped GaAs-AlGaAs SQWs, which we interpret as an excitation of the spin-split ground state of the 2DHS. We observe a doublet structure of the R-SDE, which is most pronounced if the polarization of the incoming laser is parallel to the [110] in-plane direction. Calculations confirm the interpretation that the doublet structure is a fingerprint of the in-plane anisotropy of the spin-split hole dispersion. Moreover, the experiments seem to indicate the twofold symmetry of constant-energy contours of holes in noninversion-symmetric host materials.

We acknowledge financial support by the DFG via projects SPP 1285 and SFB 689. Stefan Birner is gratefully acknowledged for technical support concerning Nextnano ${ }^{3}$.

*christian.schueller@physik.uni-regensburg.de.

[1] Semiconductor Spintronics and Quantum Computation, edited by D. D. Awschalom, D. Loss, and N. Samarth, Nanoscience and Technology (Springer, Berlin 2002); I. Zutic, J. Fabian, and S. Das Sarma, Rev. Mod. Phys. 76, 323 (2004), and references therein.

[2] M. Syperek, D. R. Yakovlev, A. Greilich, J. Misiewicz, M. Bayer, D. Reuter, and A. D. Wieck, Phys. Rev. Lett. 99, 187401 (2007).

[3] M. Kugler, T. Andlauer, T. Korn, A. Wagner, S. Fehringer, R. Schulz, M. Kubova, C. Gerl, D. Schuh, W. Wegscheider, P. Vogl, and C. Schüller, Phys. Rev. B 80, 035325 (2009).

[4] T. Korn, M. Kugler, M. Griesbeck, R. Schulz, A. Wagner, M. Hirmer, C. Gerl, D. Schuh, W. Wegscheider, and C. Schüller, New J. Phys. 12, 043003 (2010).

[5] J. P. Eisenstein, H. L. Störmer, V. Narayanamurti, A. C. Gossard, and W. Wiegmann, Phys. Rev. Lett. 53, 2579 (1984).

[6] G. Dresselhaus, Phys. Rev. 100, 580 (1955).

[7] Y. A. Bychkov and E. I. Rashba, Pis'ma Zh. Eksp. Teor. Fiz. 39, 66 (1984) [JETP Lett. 39, 78 (1984)].

[8] R. Winkler, D. Culcer, S. J. Papadakis, B. Habib, and M. Shayegan, Semicond. Sci. Technol. 23, 114017 (2008).

[9] R. Winkler, Spin-Orbit Coupling Effects in 2D Electron and Hole Systems (Springer, Berlin, 2003), and references therein.

[10] See, e.g., C. Schüller, Inelastic Light Scattering of Semiconductor Nanostructures (Springer, Berlin, 2006), and references therein.

[11] A. Pinczuk, D. Heiman, R. Sooryakumar, A. C. Gossard, and W. Wiegmann, Surf. Sci. 170, 573 (1986).

[12] M. Kirchner, C. Schüller, J. Kraus, G. Schaack, K. Panzlaff, and G. Weimann, Phys. Rev. B 47, 9706 (1993). 
[13] C. Schüller, J. Kraus, G. Schaack, G. Weimann, and K. Panzlaff, Phys. Rev. B 50, 18387 (1994).

[14] B. Jusserand, D. Richards, H. Peric, and B. Etienne, Phys. Rev. Lett. 69, 848 (1992).

[15] D. Richards, B. Jusserand, H. Peric, and B. Etienne, Phys. Rev. B 47, 16028 (1993).
[16] S. Birner, T. Zibold, T. Andlauer, T. Kubis, M. Sabathil, A. Trellakis, and P. Vogl, IEEE Trans. Electron Devices 54, 2137 (2007).

[17] C. Schüller, J. Kraus, V. Latussek, G. Schaack, and J. K. Ebeling, Solid State Commun. 81, 3 (1992).

[18] F. A. Blum, Phys. Rev. B 1, 1125 (1970). 